Provided for non-commercial research and education use. Not for reproduction, distribution or commercial use.

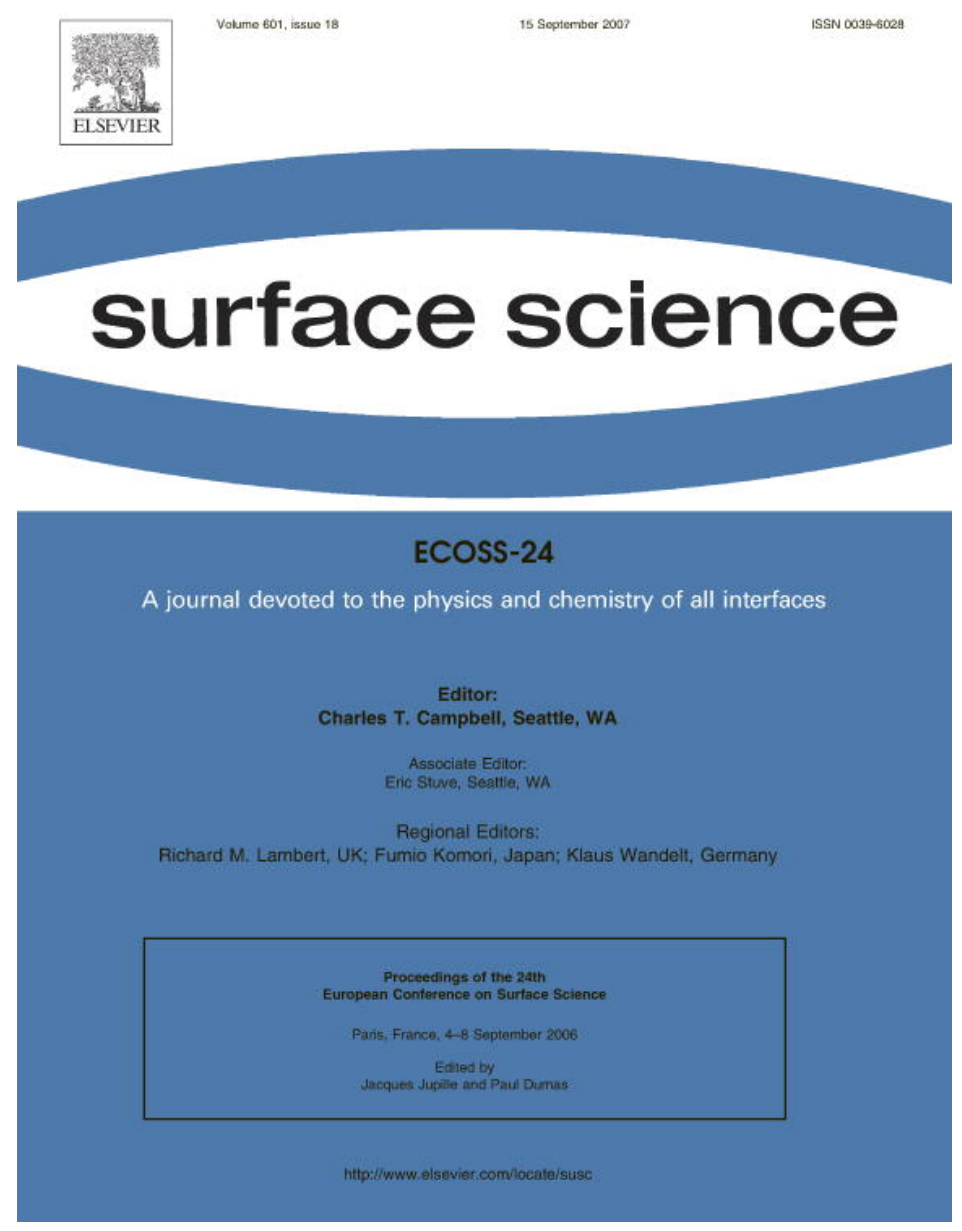

This article was published in an Elsevier journal. The attached copy

is furnished to the author for non-commercial research and education use, including for instruction at the author's institution, sharing with colleagues and providing to institution administration.

Other uses, including reproduction and distribution, or selling or licensing copies, or posting to personal, institutional or third party websites are prohibited.

In most cases authors are permitted to post their version of the article (e.g. in Word or Tex form) to their personal website or institutional repository. Authors requiring further information regarding Elsevier's archiving and manuscript policies are encouraged to visit:

http://www.elsevier.com/copyright 


\title{
Fe-doped $\mathrm{TiO}_{2}$ thin films
}

\author{
Diana Mardare ${ }^{\mathrm{a}, *}$, Valentin Nica ${ }^{\mathrm{a}}$, Cristian-Mihail Teodorescu ${ }^{\mathrm{b}}$, Dan Macovei ${ }^{\mathrm{b}}$ \\ a Alexandru Ioan Cuza University, Faculty of Physics, Carol I Blvd., No. 11, Iasi 700506, Romania \\ b National Institute of Materials Physics, P.O. Box MG-7, Atomistilor 105bis, 077125 Magurele, Romania
}

Available online 22 April 2007

\begin{abstract}
The reactive sputtering technique was used to obtain undoped and $\mathrm{Fe}$-doped $\mathrm{TiO}_{2}$ thin films deposited on glass substrates. At $250{ }^{\circ} \mathrm{C}$ substrate temperature, undoped $\mathrm{TiO}_{2}$ films crystallize in a mixed rutile/anatase phase, while Fe-doped films exhibit the rutile phase only. Presence of $\mathrm{Fe}^{3+}$ ions into the $\mathrm{TiO}_{2}$ lattice is suggested by the intensity variation of forbidden $1 \mathrm{~s} \rightarrow 3 \mathrm{~d}$ transitions between the Ti and $\mathrm{Fe}$ K-edges. Ti K-edge EXAFS data are assessed to a mixture of the two kinds of surroundings, a rutile-like crystalline phase, identified also by X-ray diffraction, and a nanosized or amorphous anatase-like surrounding. The local atomic order about Fe atoms is quite different and could be related also to an amorphous phase. The Swanepoel method is used to obtain the dispersion of the refractive index below the interband absorption edge. The dispersion energy, the single-oscillator energy and the coordination number of the Ti atoms are evaluated using the single-oscillator model (Wemple-DiDomenico).

(C) 2007 Elsevier B.V. All rights reserved.
\end{abstract}

Keywords: Thin films; Titanium oxide; Sputtering; EXAFS; XANES

\section{Introduction}

Titanium is a $3 \mathrm{~d}$ transition metal, whose oxides, in thin films, show attractive properties: high transmittance in the visible spectral range, high refractive index, high chemical and thermal durability in hostile environments, etc. Therefore, $\mathrm{TiO}_{2}$ films have a high potential for device applications. One way of controlling its properties is by doping with different atoms. In our previous papers [14] we have reported the influence of doping with $\mathrm{Ce}$, $\mathrm{Nb} \mathrm{Cr}$ or $\mathrm{Fe}$ on the structure, microstructure and, as a consequence, on the optical and electrical properties of titanium oxide films. In this paper we focused on the influence of Fe-doping on the structural properties, local atomic environment, and optical constants of titanium dioxide films.

\footnotetext{
* Corresponding author. Tel.: +40 232 201169; fax: +40 232201150.

E-mail address: dianam@uaic.ro (D. Mardare).
}

\section{Experimental}

Titanium oxide films were prepared by reactive radiofrequency (RF) sputtering in a conventional $13.56 \mathrm{MHz}$ setup (Huettinger, model PFG 300RF). The films were deposited onto glass substrates, at $250^{\circ} \mathrm{C}$. Fe-doping was achieved using a mosaic structure of $\mathrm{Ti}$ and $\mathrm{Fe}_{2} \mathrm{O}_{3}$. Oxygen $(99.998 \%)$ was used as reactive gas and argon $(99.9997 \%)$ as sputtering gas. The total pressure of the $\mathrm{Ar}+\mathrm{O}_{2}$ mixture gas during deposition was set at $0.10 \mathrm{~Pa}$, the partial pressure of $\mathrm{O}_{2}$ being of $0.03 \mathrm{~Pa}$.

For determining the phase composition of the films, $\mathrm{X}$-ray diffraction (XRD) measurements were performed at grazing incidence $\left(4^{\circ}\right)$ using $\mathrm{CuK}_{\alpha}$ radiation $(\lambda=$ $1.54178 \AA$ ).

Extended X-ray absorption fine structure (EXAFS) measurements at the $\mathrm{Ti}$ and $\mathrm{Fe} \mathrm{K}$-edges were performed on beamline E4 (station EXAFS II) at the Doris storage ring facility in Hasylab, Hamburg. Measurements were recorded in fluorescence mode, by scanning the photon energy and recording the fluorescence emitted by the 
absorbing atom of interest ( $\mathrm{Ti}$ or $\mathrm{Fe}$ ), using a standard liquid nitrogen cooled Ge detector.

The optical transmittance of the films was measured in the visible region using a SPECORD UV-VIS doublebeam spectrophotometer, Carl Zeiss Jena.

\section{Results and discussion}

XRD studies performed on several sets of undoped and Fe-doped $\mathrm{TiO}_{2}$ samples deposited onto glass substrates, reveal that a phase transformation (anatase to rutile) take place by iron doping films $[3,5]$. The weight percentage of the rutile phase among the total crystallized phase (i.e. by excluding amorphous contributions which do not reflect in XRD), as calculated using a formula proposed by Spurr and Myers [6], increases by doping from 30\% to $100 \%$ [5].

Based on EDX measurements [5], the chemical formula of Fe-doped sample may be approximated as $\mathrm{Fe}_{0.01} \mathrm{Ti}_{0.99} \mathrm{O}_{2}$.

We denote by $\mathrm{S}$ and $\mathrm{S}$.Fe the undoped and Fe-doped sample, respectively. The anatase average grain size, obtained from A(101) peak via Scherrer's formula, is about $16 \mathrm{~nm}$, while the rutile peak $\mathrm{R}(110)$ is wide for both samples, indicating that rutile grain size is about $7 \mathrm{~nm}$.

Fig. 1 shows the X-ray absorption spectra recorded for Fe-doped sample, S.Fe, at the Ti K-edge (a) and at the Fe K-edge (b). Inserts in both figures present the pre-edge region and the near-edge absorption structure (XANES) at the corresponding edges.

The pre-edge peaks A (Fig. 1) are ascribed to dipole forbidden transitions on $4 \mathrm{~s}$ and mainly $3 \mathrm{~d}$ states and their aspect is different for absorbing atoms in different coordination [7]. At the same time, the Fe K-edge XANES shows a pre-edge peak of considerable less intensity. The same peak of less intensity occurs also in the Fe K-edge spectrum of hematite $\mathrm{Fe}_{2} \mathrm{O}_{3}$, which was measured as a standard by using the same experimental setup. Its reduced intensity is also a sign of the reduced number of $3 \mathrm{~d}$ vacancies on which one may excite the core electron (as compared with this value for $\mathrm{Ti}$ ). We estimated the sole integral (calculated in [arbitrary units of absorption $\times \mathrm{eV}$ ]) of each pre-edge peak, normalized to the intensity of the absorption jump (in [arbitrary units of absorption]). The obtained values are $0.42 \mathrm{eV}$ for $\mathrm{Ti}$ K-edge in $\mathrm{TiO}_{2}$, $0.20 \mathrm{eV}$ for both Fe K-edge in hematite and for Fe K-edge in $\mathrm{TiO}_{2}: \mathrm{Fe}$. By comparison of the integral intensity between $\mathrm{Fe}$ and $\mathrm{Ti}$ pre-edge peaks and by considering only dipole forbidden transitions on $3 \mathrm{~d}$ states, with $3 \mathrm{~d}^{0}$ configuration for $\mathrm{Ti}^{4+}$ in titania, one may infer a configuration close to $3 \mathrm{~d}^{5}$ for $\mathrm{Fe}$ in both hematite and in $\mathrm{TiO}_{2}: \mathrm{Fe}$. This means that iron atoms in the sample of interest $\mathrm{TiO}_{2}: \mathrm{Fe}$ are in a $3+$ ionization state. The similarity of these two iron states is also supported by the similitude of the overall aspects of the corresponding XANES spectra.

The peak $\mathrm{B}$ is a sign also of octahedral ionic cage, whereas peak $\mathrm{C}$ can be regarded as a resonance connected to excitation with charge transfer on second-order cationic neighbors, at least for zincblende structure [8]. The pres-
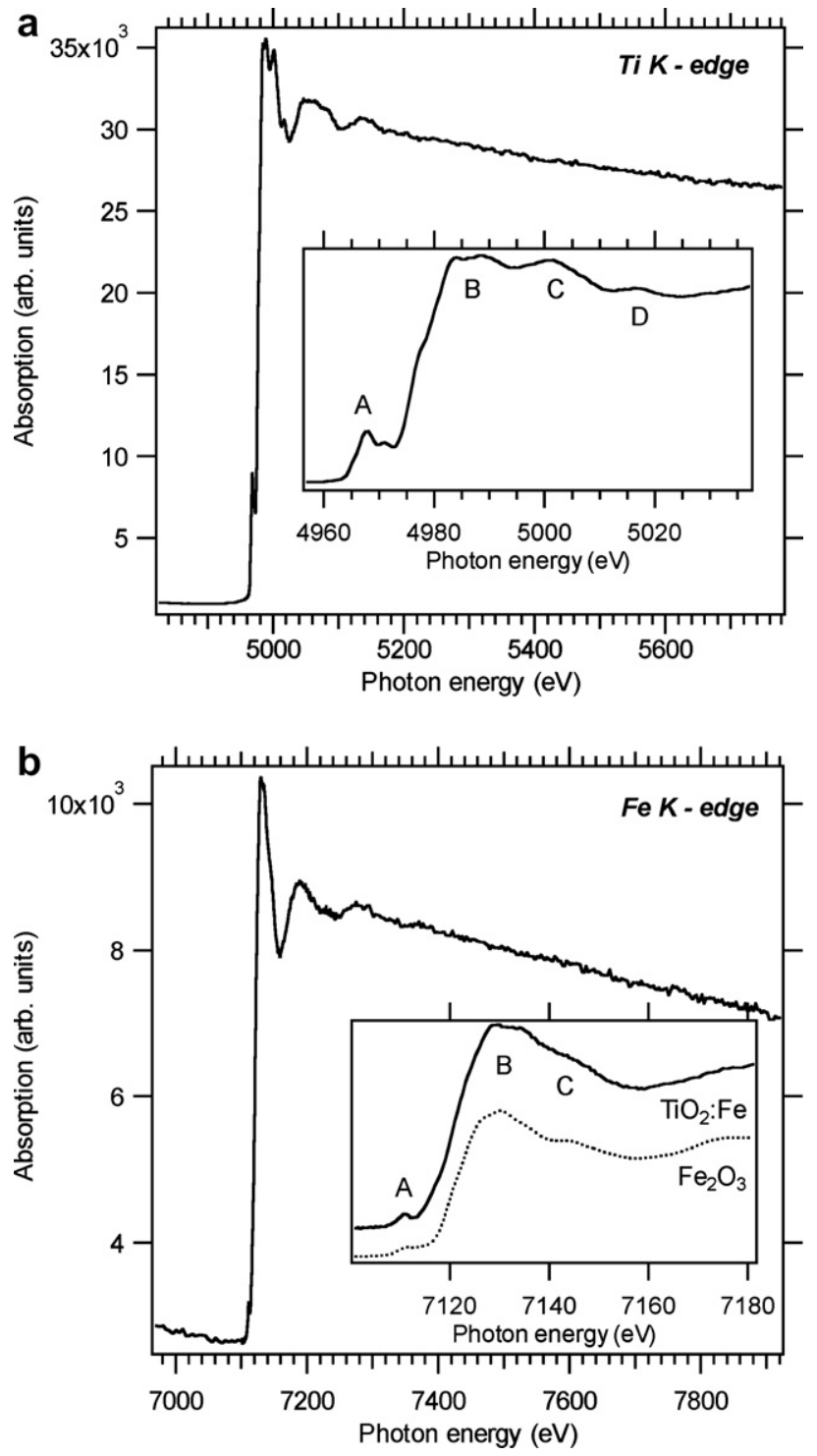

Fig. 1. Absorption spectra (including pre-edge, XANES and EXAFS regions) at Ti K-edge (a) and Fe K-edge (b) in iron-doped titania. Inserts: the pre-edge and XANES regions of the two spectra. In the insert of (b) also the Fe K-edge XANES of pure hematite $\left(\mathrm{Fe}_{2} \mathrm{O}_{3}\right)$, recorded in the same experimental setup, is shown.

ence of peak B and the considerable decreased intensity of peak $\mathrm{C}$ in the Fe XANES may be viewed as a sign of a less defined long-range order in the $\mathrm{Fe}$ environment. It may be possible that a strong distortion of the octahedral lattice, as derived from peak $\mathrm{B}$, to be accompanied by a less effective mechanisms of excitation with charge transfer (peak C).

Peak D is a multiple scattering XANES resonance in titania [8,9] and its absence at the Fe edge may also be viewed as a sign of local amorphisation around the Fe ions.

When analyzing Ti K-edge EXAFS data, we could infer that the local atomic order around Ti atoms is not similar to pure rutile nor to pure anatase, but rather to a mixture of the two kinds of surroundings (Ti1 and Ti2). The first Ti neighbouring is assessed by taking into account that X-ray 
diffraction has identified rutile crystals (Ti1); consequently by comparison with the Ti K-edge EXAFS data, the occurrence of another amorphous or nanosized phase with anatase-like surrounding (Ti2) is proposed. Dopant segregation was pushed back, since the EXAFS spectrum of such segregated structures is quite different [10]. Concerning the $\mathrm{Fe} \mathrm{K}$-edge EXAFS data, this is more similar to the anatase-like environment [9], so we infer that Fe atoms occupy sites which are quite similar to the amorphous or nanosized anatase-like environment of Ti2, in a phase which did not exhibit long-range ordering.

The recorded EXAFS spectra, after standard data treatment [11] are represented in Fig. 2. For computation of the photoelectron wavevector, edge energies before the onset of the pre-edge peak (4965 eV for $\mathrm{Ti}, 7109.5 \mathrm{eV}$ for $\mathrm{Fe}$ ) have been considered instead of inflection points of the main edge. This choice leads to a much better match between the recorded EXAFS spectra and the simulated EXAFS by using the anatase or rutile structure. The simulations are also shown in Fig. 2. In computing these simulations, the complete anatase and rutile structures have been considered, together with values of the Debye-Waller factors of $0.15 \AA$ and the inelastic mean free path dependence obtained from [12]. The backscattering amplitudes, backscattering phase shifts and absorbing atom phase shifts were taken from Ref. [11]. The obtained simulated spectra are similar to the measured spectra reported recently in [13];

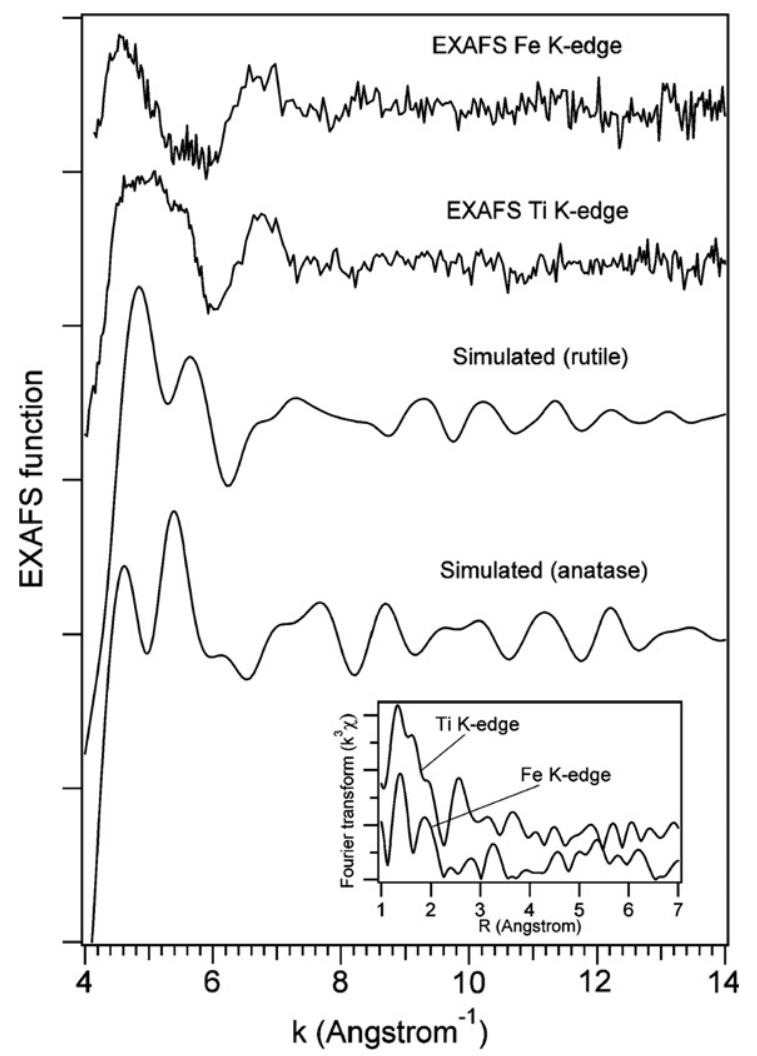

Fig. 2. Extracted $\mathrm{Ti}$ K-edge and Fe K-edge EXAFS spectra from absorption spectra given in Fig. 1 together with simulated anatase and rutile EXAFS spectra (see text for details). Insert: Fourier transforms of the experimental EXAFS spectra. the discrepancies can be related to multiple scattering effects [9]. Also, in the insert of Fig. 2 we have represented the Fourier transform of the EXAFS spectra, both at $\mathrm{Ti}$ and Fe K-edges.

A direct comparison between the measured Ti EXAFS spectra and the simulated ones can be used as an evidence of co-existence of rutile and anatase-like surrounding in the sample. A least square fit refinement of the first features led to a relative contribution of $62 \pm 8 \%$ anatase-like to the total spectrum. We cannot speculate more on this aspect since it seems that titania phases with strongly distorted octahedral structures are present in the EXAFS spectrum. Taking into account X-ray diffraction data, these phases are amorphous. Note also, when speaking about anataselike environment that the Fourier transform of the spectrum is not quite similar to the anatase one [13], especially concerning local atomic environment at the 3rd and 4th coordination shell. A further refinement of the EXAFS spectra is a complicated work and efforts are in course to extract maximum of information from these spectra.

The EXAFS at the Fe K-edge is considerably different from the EXAFS at the Ti K-edge. From the Fourier transform of the EXAFS spectrum at the Fe K-edge (insert in Fig. 2) one may infer the presence of two well defined coordination shells at low interatomic distances about the $\mathrm{Fe}$ atoms, whereas in the local environment of Ti contributions from several shells with slightly different interatomic distances are merged into the first peak of the Fourier transform. For larger interatomic distances, the radial distribution function about the Fe atoms decrease drastically, which can be seen as a sign of a lower long-range order about these atoms.

The wavelength dependency of the films refractive index, $n(\lambda)$, has been examined by using the Swanepoel method [14]. This method needs the optical transmittance spectra for the studied thin films. $\mathrm{TiO}_{2}$ films are transparent in the visible region [3] and its transparency exhibits a sharp decrease in the UV region. The fluctuations on these spectra are connected with the film thickness and consequently with the interference in the layer. The number of the available fringes gives the accuracy of this method [14]. The refractive index of the substrate in the region of weak absorption was determined by means of transmission spectrum for the clean substrate alone and it was found to be 1.47. The curves $n(\lambda)$, presented in Fig. 3, show a Sellmeier dispersion type dependence as reported in literature [4] for the visible spectral range, where $\mathrm{TiO}_{2}$ films are transparent.

The oscillator energy and strength and the optical bandgap can be determined by applying the WempleDiDomenico relation [15]

$n^{2}(v)=1+\frac{E_{\mathrm{o}} E_{\mathrm{d}}}{E_{\mathrm{o}}^{2}-(h v)^{2}}$

where $E_{\mathrm{o}}$ and $E_{\mathrm{d}}$ represent the oscillator energy and dispersion energy (the strength of interband optical transitions) respectively and $h v$ is the photon energy. 


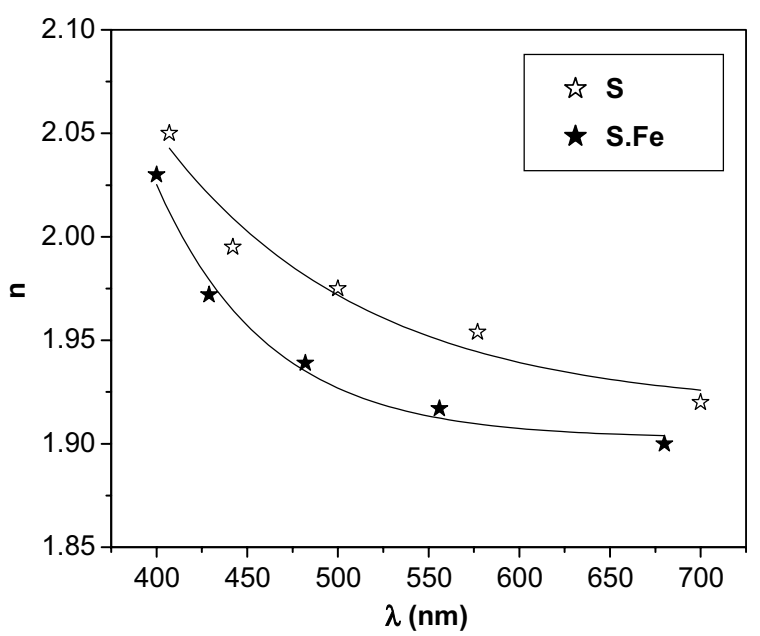

Fig. 3. The refractive index $n$ versus wavelength $\lambda$, in the visible range, for undoped and $\mathrm{Fe}$-doped $\mathrm{TiO}_{2}$ films.

By putting the relation (1) in the form

$\frac{1}{n^{2}-1}=\frac{E_{\mathrm{o}}}{E_{\mathrm{d}}}-\frac{(h v)^{2}}{E_{\mathrm{o}} E_{\mathrm{d}}}$

and representing $\left(n^{2}-1\right)^{-1}$ versus $(h v)^{2}$, a linear behavior is obtained (Fig. 4) having correlation coefficients very close to $1(0.999)$. The slope of the linear dependence is $\left(E_{\mathrm{o}} E_{\mathrm{d}}\right)^{-1}$, and the intercept with the vertical axis, $E_{\mathrm{o}} / E_{\mathrm{d}}$. The oscillator energy, $E_{\mathrm{o}}$, is an "average" energy gap, as specified in the literature $[15,16]$. For the undoped film, the calculated values of $E_{\mathrm{d}}$ and $E_{\mathrm{o}}$ are $16.72 \mathrm{eV}$ and $6.67 \mathrm{eV}$, respectively, while for the Fe-doped film these values are $15.85 \mathrm{eV}$ and $6.64 \mathrm{eV}$, respectively.

Wemple has established an empirical relation for the dispersion energy

$E_{\mathrm{d}}=\beta N_{\mathrm{c}} N_{\mathrm{e}} Z_{\mathrm{a}}$

where $N_{\mathrm{c}}$ is the coordination number of the cation that is the nearest neighbor to the anion, $N_{\mathrm{e}}$ is the effective num-

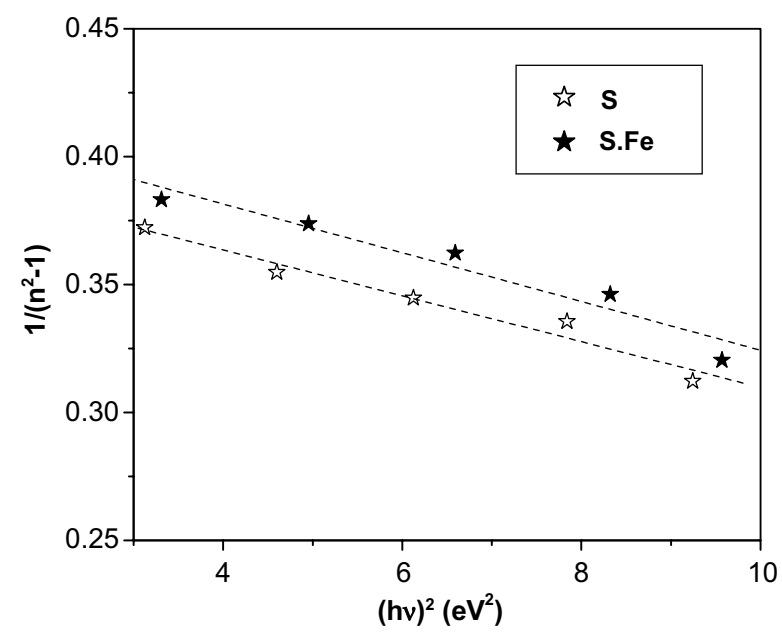

Fig. 4. $\left(n^{2}-1\right)^{-1}$ versus $(h v)^{2}$, in the visible range, for undoped (sample S) and $\mathrm{Fe}$-doped (sample S.Fe) $\mathrm{TiO}_{2}$ films. The dash lines represent the fitting straight-lines. ber of valence electrons per anion, $\beta$ is a constant and $Z_{\mathrm{a}}$ is the formal chemical valence of the anion. For $\mathrm{TiO}_{2}$, $\beta \cong 0.22[15], N_{\mathrm{e}}$ has the value 8 , as already specified in Ref. [4] and $Z_{\mathrm{a}}=2$. From relation (3), the values of $N_{\mathrm{c}}$ are 4.75 and 4.5 for $S$ and S.Fe, respectively.

A rutile sample $(\mathrm{S} . \mathrm{Fe})$ is expected to be more dense than the mixed anatase/rutile sample (S) and to have higher refractive indices. The calculated $n$ values presented in Fig. 3 do not confirm this observation. In a qualitative sense, the refractive index is the "optical density" of a material and the mentioned discrepancies can be related to the fact that the doped sample may have a porosity that would lower the refractive index [3]. Here, not only the phase composition brings its contribution, but also the fact that the sample S.Fe has nanosized grains [5]. The values of $n(633 \mathrm{~nm})(1.93$ in the undoped sample and 1.90 in Fedoped sample), much smaller than the average $n$ values of rutile and anatase single crystals (2.75 and 2.52, respectively [17]), prove a high void content $(>5 \%)$ in the films. Consequently, the structural differences together with the microstructural ones, explain why the Fe-doped $\mathrm{TiO}_{2}$ films, with a rutile phase spread in an amorphous phase, exhibit lower $n$ values, as compared to the undoped $\mathrm{TiO}_{2}$ film (Fig. 4). According to Ref. [4], an XRD amorphous sample is characterized by lower refractive indices than the polycrystalline ones and it has also lower dispersion energy and coordination number of the cation. Here, for the nanostructured sample S.Fe we reach to the same conclusion.

\section{Conclusions}

Reactive RF sputtering was utilized to deposit undoped and $\mathrm{Fe}$-doped $\mathrm{TiO}_{2}$ thin films onto glass substrates. When deposited onto heated substrates, the films present polycrystalline structures. By doping with low concentrations of Fe, the structure changes from mixed anatase/rutile to pure rutile.

From Fe K-edge extended X-ray absorption fine structure, some general conclusions may be summarized as follows: (i) $\mathrm{Fe}$ atoms are in 3+ ionization states; (ii) the long-range order about the $\mathrm{Fe}$ atoms is considerably affected, as compared with that around the Ti atoms (from both XANES and EXAFS data); (iii) the Ti EXAFS spectrum reveals a mixture of rutile phase (Til) and of an amorphous phase where $\mathrm{Ti}$ atoms are in locally distorted anatase-like environment (Ti2), but with no long-range ordering visible by X-ray diffraction; (iv) the edge energy had to be chosen just at the onset of the pre-edge peak instead of on the main edge for both $\mathrm{Fe}$ and $\mathrm{Ti}$.

The dispersion of the refractive index below the interband absorption edge was obtained by applying the Swanepoel method to optical transmittance spectra. The dispersion energy and the single-oscillator energy in the undoped film are $16.72 \mathrm{eV}$ and $6.67 \mathrm{eV}$, respectively, while in the Fe-doped film these values are $15.85 \mathrm{eV}$ and $6.64 \mathrm{eV}$, respectively. The structural differences together with the microstructural ones, explain why the Fe-doped $\mathrm{TiO}_{2}$ films, 
with a single rutile phase spread in an amorphous phase, exhibit lower $n$ values, and also lower values for the coordination number compared to the undoped $\mathrm{TiO}_{2}$ film.

\section{Acknowledgements}

This work was supported by the Romanian Ministry of Education and Research through Contract CNCSIS 1148/ 2006 and Contract CEEX-Matnanech No. 23/2005. EXAFS experiments were possible through the long-term Hasylab project II-05-058. We acknowledge qualified support from Dr. Edmund Welter and Dr. Dariusz Zajac from the Hasylab staff, and scientific cooperation from Prof. Eberhardt Burkel and Dr. Radu Nicula from the University of Rostock in setting up the Hasylab proposal.

\section{References}

[1] D. Luca, D. Mardare, F. Iacomi, C.M. Teodorescu, Appl. Surf. Sci. 252 (2006) 6122.

[2] D. Mardare, G.I. Rusu, F. Iacomi, M. Girtan, I. Vida-Simiti, Mater. Sci. Eng. B 118 (1-3) (2005) 187.

[3] D. Mardare, M. Tasca, M. Delibas, G.I. Rusu, Appl. Surf. Sci 156 (2000) 200.

[4] D. Mardare, P. Hones, Mater. Sci. Eng. B 68 (1999) 42.
[5] D. Mardare, F. Iacomi, D. Luca, Thin Solid Films 515 (2007) 6474.

[6] R.A. Spurr, H. Myers, Anal. Chem. 29 (1957) 760.

[7] S. Eiden-Assmann, J. Widoniak, H. Huwe, M. Fröba, Hasylab annual report 2003, <http://www-hasylab.desy.de/science/annual reports/2003_report/index.html $>$.

[8] C.M. Teodorescu, J.M. Esteva, M. Womes, A. El Afif, R.C. Karnatak, A.M. Flank, P. Lagarde, J. Electron Spectrosc. Relat. Phenom 106 (2000) 233.

[9] M.F. Ruiz-Lopez, A. Munoz-Paez, J. Phys. Condens. Mat. 3 (1991) 8981;

F. Bohr, M.F. Ruiz-Lopez, A. Munoz-Paez, Catal. Lett. 20 (1993) 59.

[10] R. Bouchet, A. Weibel, P. Knauth, G. Mountjoy, A.V. Chadwick, Chem. Mater. 15 (2003) 4996.

[11] B.K. Teo, EXAFS: Basic Principles and Data Analysis, Springer, Berlin, 1983.

[12] S. Hüfner, Photoelectron Spectroscopy: Principles and Applications, Springer, Berlin, 2003.

[13] I. Schlipf, M. Bauer, G. Kickelbick, H. Bertagnolli, Hasylab annual report, 2005, p. 333, <http://www-hasylab.desy.de/science/annual_ reports/2005_report/index.html $>$.

[14] R. Swanepoel, J. Phys. E: Sci. Instrum. 16 (1983) 1214.

[15] S.H. Wemple, M. DiDomenico, Phys. Rev. B 3 (1971) 1338.

[16] E. Márquez, J.B. Ramirez-Malo, P. Villares, R. Jiménez-Garay, R. Swanepoel, Thin Solid Films 254 (1995) 83.

[17] CRC Handbook of Chemistry and Physics, 76th ed., CRC Press, Boca Raton, FL, 1995 (p. 4, 137). 